\title{
Formación de ciudadanos ambientales mediante un curso sobre la sostenibilidad del agua utilizando TIC
}

\author{
Estefania Ruiz-Barrios \\ Maestría en Educación para la Ciudadanía, Facultad de Psicología, Universidad \\ Autónoma de Querétaro. Querétaro. México.psic.estefania.rb@gmail.com \\ ORCID: https://orcid.org/0000-0001-6902-4922 \\ María Evelyn Diez-Martínez Day \\ Maestría en Educación para la Ciudadanía, Centro de Investigación en Tecnología \\ Educativa, Facultad de Psicología Universidad Autónoma de Querétaro. México. \\ evelyn@uaq.mx \\ ORCID: https://orcid.org/0000-0002-8521-4731
}

[Recibido: 3 septiembre 2020. Revisado: 13 octubre 2020. Aceptado: 16 enero 2021]

Resumen: Una de las problemáticas actuales es el futuro comprometido del agua, en términos de su sostenibilidad. La educación para la ciudadanía ambiental no ha logrado los impactos esperados en los cambios de hábitos y consumo de la población con relación a este imprescindible recurso. Innovaciones en la educación, que retomen o promuevan el interés en los jóvenes sobre esta problemática, son necesarias ya que son las generaciones actuales y futuras las que tendrán que enfrentar los retos al respecto. El uso generalizado de estas poblaciones de la tecnología hace necesario probar el impacto de su utilización para la educación ambiental y la concientización de la participación en el uso y consumo del agua. Esta investigación tuvo como objetivo principal determinar los efectos de la implementación de un curso sobre el tema de la sostenibilidad del agua mediante el uso de herramientas digitales en la educación para la ciudadanía ambiental con estudiantes de una universidad pública en México. Esta investigación retomó la participación de 26 estudiantes para analizar el efecto que produjo en ellos la reflexión, análisis, crítica y diálogo sobre actividades humanas relacionadas con el agua y su importancia vital con el ambiente, mediante una concepción holística. Los temas tratados incluyeron: 1. Elementos conceptuales sobre el agua, 2. El agua y su importancia desde un enfoque holístico, 3. Situaciones y problemas del agua, y 4 . Acciones como ciudadanos ambientales.

Los resultados obtenidos indican que hubo un incremento del conocimiento, sensibilización y comportamiento pro ambiental ante los temas revisados, ello al comparar los resultados obtenidos en la evaluación Pre-Test y Post-Test, donde el cambio de su percepción hacia el agua y el ambiente fue considerable. Finalmente, la discusión de los resultados retomó aspectos teóricos e investigaciones sobre Educación Ambiental, Psicología Ambiental y Ciudadanía Ambiental.

Palabras clave: Sostenibilidad del agua; Educación Ambiental; Ciudadanía Ambiental; TIC; Universitarios.

\section{Training of environmental citizens through a course on water sustainability using ICT}

Abstract: One of the current problems is the compromised future of water, in terms of its sustainability. Education for environmental citizenship has not achieved the expected impacts on the changes in habits and consumption of the population concerning this essential resource. Innovations in education that resume or promote interest in young people on this problem are necessary since it is the current and future generations who will have to face the challenges in this regard. The widespread use of technology by these population makes it necessary to test the impact of its use for environmental education and awareness of participation in the use and consumption of water. The main objective of this research was to determine the effects of the implementation of a course on the subject of water sustainability through the use of digital tools in education for environmental citizenship in students of a public university in Mexico. Thus, this research took up the participation of 26 students to analyze the effect that reflection, analysis, criticism and dialogue on human activities 
related to water and the vital importance of this resource for the environment produced on them through a holistic conception. The topics covered included: 1. Conceptual elements about water, 2. Water and its importance from a holistic approach, 3. Situations and problems of water, and 4. Actions as environmental citizens.

The results obtained indicate that there was an increase in knowledge, awareness and proenvironmental behavior regarding the reviewed topics, this when comparing the results obtained in the Pre-Test and Post-Test evaluation, where the change in their perception towards water and the environment was considerable. Finally, in the discussion of the results, theoretical aspects and research aspects concerning Environmental Education, Environmental Psychology and Environmental Citizenship.

Keywords: Water sustainability; Environmental Education; Environmental Citizenship; ICT; University students.

Para citar este artículo: Ruiz-Barrios, E. y Diez-Martínez, M. E. (2021) Formación de ciudadanos ambientales mediante un curso sobre la sostenibilidad del agua utilizando TIC. Revista de Educación Ambiental y Sostenibilidad 3(1), 1301. doi: 10.25267/Rev_educ_ambient_sostenibilidad.2021.v3.i1.1301

\section{Introducción}

El agua es un recurso fundamental y esencial para el desarrollo, funcionamiento y conservación de la vida en el planeta. El planeta cuenta solo con un $2.5 \%$ de agua dulce, que es la única apta para el consumo humano. Casi el 30\% de este porcentaje es agua subterránea y de difícil acceso; casi el 70\% se encuentra en glaciares y capas polares y el, aproximadamente, 1\% se encuentra en ríos, lagos y en la atmósfera. Al ser un recurso tan escaso para el consumo ocasiona que el $11 \%$ de la población total mundial no tenga acceso al agua potable, encontrando que en Latinoamérica 32 millones de personas no tengan acceso al agua, basado en el informe de SEMARNAT/CONAGUA de 2011 (Consejo Consultivo del Agua, A. C., 2018a). Esta situación se ha incrementado al pasar de los años, encontrando tres factores que producen escasez del agua: a) el crecimiento demográfico, b) el aumento del consumo de agua en el sector industrial y, c) la expansión del cultivo de regadío del sector agrícola, (Cervantes-Jiménez, 2017, p. 17), por lo que su descuido y su uso excesivo para satisfacer necesidades humanas directa o indirectamente ha propiciado su escasez.

Por su parte, México únicamente posee el $0.1 \%$ de agua dulce a nivel mundial, por lo que se cataloga como un territorio semidesértico (Fondo para la comunicación y la educación ambiental, A. C., 2018). Pese a que;

desde hace años el gobierno y los medios han señalado en repetidas ocasiones que el agua es cada vez más escasa, al parecer, aún no se toma conciencia del problema, pues las personas, cuando abren el grifo, no toman en cuenta lo escaso del recurso ni lo que cuesta, o todo lo que implica abastecer a las ciudades (Esparza, 2014, p. 195)

Esta falta de conciencia ha llevado a que cuatro de cada diez personas en México no tengan acceso al agua (Consejo Consultivo del Agua A. C., 2018b). La falta de acceso al agua en la mayoría de la población ha producido que esta situación se considere cotidiana y no genere conciencia o sensibilización sobre su cuidado y escasez.

Esta investigación buscó hacer conciencia y sensibilización sobre la importancia del agua de manera holística (biofísica, económica, política, ambiental, cultural y psicológica). La investigación se realizó en el estado semidesértico de Querétaro, 
específicamente, en la ciudad de Querétaro, que posee aproximadamente 2 millones de habitantes y que cuenta con un gran crecimiento poblacional e industrial, lo que ha provocado un gran estrés hídrico (INEGI, 2020). La investigación tuvo como objetivo contribuir a la formación de ciudadanos ambientales a través de un curso mediante herramientas digitales, fomentando el análisis, reflexión, sensibilización y concientización del cuidado, uso y respeto al agua.

\section{Educación Ambiental y Ciudadanía Ambiental}

Desde el ámbito educativo, la Educación Ambiental ha tenido como uno de sus principales objetivos «la formación de los futuros ciudadanos y ciudadanas y hacer comprender la necesidad de acciones que contribuyan a un futuro sostenible en los diferentes ámbitos: consumo responsable, actividad profesional y acción ciudadana» (Vilches y Gil-Pérez, 2010, p. 306). Tal objetivo se ha visto limitado o inconcluso en la práctica del área educativa ya que, como Vilches y Gil-Pérez (2010) señalan, los educadores en general no están prestando suficiente atención a la situación a pesar de los llamamientos de Naciones Unidas en las Cumbres de La Tierra (Río 1992; y Johannesburgo, 2002). Algunos autores (Diez-Martínez, 2014; Kollmuss y Agyeman, 2002) han señalado que la educación sobre el ambiente ha estado incorporada en los currículos educativos de la mayoría de los países del mundo desde hace varias décadas y, sin embargo, no ha habido efecto en el cambio de hábitos del ciudadano por evitar el deterioro de recursos naturales y socioculturales.

Esta situación se percibe desde la educación básica hasta la universidad, teniendo como consecuencia, "comportamientos individuales y colectivos orientados a la búsqueda de beneficios particulares y a corto plazo, sin atender a sus consecuencias para los demás o para las futuras generaciones», (Vilches y Gil-Pérez, 2010, p. 315). Calvo, Ferreras y Rodrigo-Cano (2020) mencionan que, ante esta situación, es necesario potenciar a los estudiantes como emisores y ofrecerles estímulos, posibilidades y capacidades para la transmisión de mensajes e información sobre educación ambiental.

Por lo anterior, han surgido distintas perspectivas de la manera en que la Educación Ambiental debe ser impartida, de entre las que Sauvé (2005) propone dos clasificaciones: las corrientes con una larga tradición y las corrientes más recientes. Para esta investigación, resultan relevante, por un lado, la corriente resolutiva, perteneciente a las corrientes de una larga tradición, centrada en el estudio de problemáticas ambientales y sus componentes sociales y biofísicos, la identificación de una situación problema y realizando un diagnóstico que busque y elija soluciones óptimas, y, por otro lado, la corriente holística (que se encuentra dentro de las corrientes más recientes), y que toma en cuenta las múltiples dimensiones de las realidades socio ambientales, así como las diversas dimensiones de la persona en relación con estas realidades, es decir, la complejidad de su ser en el mundo, donde cada realidad está unida a una red de relaciones y donde todo adquiere un sentido. Gutiérrez-Yurrita (2009) menciona que el holismo surge como un nuevo paradigma capaz de romper los paradigmas reduccionistas, por lo que posibilita un nuevo lenguaje y conceptos para ver de forma nueva y diferente el todo, es decir, propone un sistema complejo como una forma nueva de cultura en donde las propiedades y leyes no pueden ser explicadas por sistemas simples. De esta manera, se busca un avance cultural para pasar de una relación de dominio y explotación del sujeto sobre el 
objeto-ambiente, a una relación de respeto e igualdad entre entes, una relación de interdependencia holística, donde todos formamos parte del ambiente, que es diferente a la suma de sus partes (Rodríguez-Peñaguirre y Rabell, 2019).

Siguiendo a Sauvé (2014), la Educación Ambiental busca construir una identidad ambiental que dé sentido a nuestro ser-en-el-mundo, desarrollar una pertenencia y promover una cultura de compromiso, que induzca dinámicas sociales que favorezcan la colaboración y el enfoque crítico de las realidades socio-ecológicas que permitan asumir de manera autónoma y creativa la resolución de problemas, así como desarrollar proyectos que contribuyan a una mejor relación con el ambiente.

Se parte de la idea de contribuir a la formación de un Ciudadano Ambiental:

[...] cuyo sentido de la responsabilidad lo lleva a cuidar los bienes colectivos de la sociedad a la que pertenece (que son los bienes inmediatos) y del resto del mundo (que son los bienes y servicios mediatos). Es sensible al deterioro ambiental, a la sobreexplotación de los recursos naturales, al abuso de tecnologías altamente demandantes de energía, a la generación inconsciente de residuos, etc., pero al mismo tiempo, también es consciente de que él forma parte del problema. De esta manera, se convierte en un individuo que, al reflexionar sobre la situación ambiental del mundo, es como si mirara en un espejo su reflejo, su propio proceder [...] (GutiérrezYurrita, 2014, p. 49)

Sumado a lo anterior, Gutiérrez-Yurrita (2009) considera como ciudadanos ambientales a aquellos que luchan por los derechos de los menos favorecidos, por la conservación de la naturaleza, por el uso de tecnologías limpias para que la globalización económica no tenga exclusivamente intereses económicos de empresas transnacionales, buscando un proceso de mundialización donde se elimine el radicalismo del capitalismo, fomentando apoyos a las comunidades locales y promoviendo valores culturales. ( $\leftarrow$ Si la cita es textual, debe marcarse como tal).

Ante el concepto de ciudadano ambiental la psicología ambiental propone aspectos que determinan el comportamiento pro ambiental, que puede catalogarse como un comportamiento protector del ambiente. Considerando la propuesta de CorralVerdugo y de Quiroz (2004), este comportamiento debe cumplir con cinco características psicológicas: efectividad, deliberación, anticipación, solidaridad y austeridad. El cual estaría indicado por un conjunto de acciones efectivas, deliberadas y anticipadas que busquen la preservación de los recursos naturales, así como buscar la integridad de todas las especies.

Por su parte, Kollmuss y Agyeman (2002) proponen que la conciencia pro ambiental posee cierta complejidad a la hora de visualizar su entendimiento, ya que integra valores personales amplios moldeando rasgos de la personalidad, así como de factores externos (sociales y culturales). Aunado a ello, Hines, Hungerford y Tomera (1986) agregan que algunos factores pueden influenciar este tipo de conductas en favor del ambiente, tales como: el conocimiento que se tiene sobre los problemas ambientales y cuáles son sus causas; conocer estrategias de acción para reducir el impacto negativo en el ambiente; tener actitudes en favor del ambiente; pronunciarse a sí mismo como un protector ambiental o tener un gran sentido de la 
responsabilidad. Así mismo, Hines, Hungerford y Tomera (1986) mencionan que existen factores situacionales que pueden fungir como limitantes en esta conducta, tales como: económicos, presiones sociales, y falta de oportunidades para llevar a cabo este tipo de acciones.

\section{TIC en la Educación Ambiental}

Las TIC dentro de la educación tienen como objetivo proporcionar información a los estudiantes, todo ello con el apoyo de profesores que posibiliten el conocimiento del entorno y sus problemas, procurando un enfoque participativo y emancipador a través de la reflexión (Carvalho de Sousa, et al., 2012).

La obtención y acceso de información a partir de la alfabetización digital en temas ambientales, ha favorecido los procesos de aprendizaje basados en problemáticas globales que pueden ser comparadas a nivel local (Ojeda-Barceló et al., 2011). Por tanto, las TIC poseen gran importancia en el momento del aprendizaje y enseñanza de los alumnos, ya que estos pueden percibir, comparar y analizar problemas ambientales en el contexto macro y micro. De esta manera, los estudiantes utilizan estos medios para acceder, interpretar y organizar la información en conocimiento al momento de pensar y proponer soluciones ante dichas problemáticas (Yildirim, 2005).

Las herramientas digitales funcionan como nuevas formas de aprendizaje, donde se posibilita la construcción de su propio conocimiento a través de medios de búsqueda, comunicación y participación en el ámbito de la Educación Ambiental, lo que permite contribuir al mejoramiento de la relación entre hombre y ambiente al buscar aspectos de la interdisciplinariedad sobre el tema «que favorezcan las actividades colaborativas, que puedan aplicarse a contextos diferentes, que no contaminen, que sean de fácil acceso, reutilizables, así como de bajo precio.» (Galindo, 2015, p. 336).

En algunos estudios anteriores sobre el efecto de cursos ligados a temáticas ambientales (Corres y Diez-Martínez, 2019; Fernández, Reyes y Piñero, 2018;) con estudiantes de nivel de bachillerato y universitario, se ha mostrado que al implementar intervenciones pedagógicas a manera de cursos sobre la promoción de conductas pro-ambientales y de consumo sostenible utilizando las TIC, se observa un incremento en el aprendizaje y motivación, generando aportes muy interesantes de los participantes sobre las problemáticas ambientales y socioculturales incluidas en los contenidos de dichos cursos.

Considerando estos antecedentes y tratando de continuar analizando las posibilidades de las TIC en la comprensión y difusión de problemáticas ambientales y que contribuyen a la formación de ciudadanos ambientales, el objetivo de este trabajo consistió en determinar los efectos de la implementación de un curso sobre el tema de la sostenibilidad del agua mediante el uso de herramientas digitales en la educación para la ciudadanía ambiental en estudiantes de una universidad pública en México.

\section{Metodología}

La investigación se realizó con una muestra intencional de 26 alumnos universitarios de la Facultad de Psicología de la Universidad Autónoma de Querétaro, México, 
quienes dieron su consentimiento informado con respecto a los alcances $\mathrm{y}$ aplicaciones de dicha investigación.

Los sujetos participantes habitan en una ciudad con el segundo lugar en crecimiento poblacional del país, y con aproximadamente 2 millones de habitantes (INEGI, 2020). Se encuentra en el altiplano mexicano donde el agua escasea y la mayoría del agua utilizada procede de pozos del subsuelo, haciendo extracción del manto freático, que según expertos puede acabarse en menos de 30 años (Diez-Martínez, 2013). Aunado a esto, la rápida expansión económica y el incremento poblacional exponencial, está generando un sinnúmero de problemáticas ambientales de las cuales es necesario concientizar a sus habitantes (Ochoa-Ramírez et al., 2019; Granados-Ramírez y Villaseñor-Franco, 2007), en particular a los jóvenes, para fomentar su participación con miras a mejorar su futuro próximo.

La realización de la investigación constó de cuatro fases que contribuyeron a realizar el curso (Figura 1).

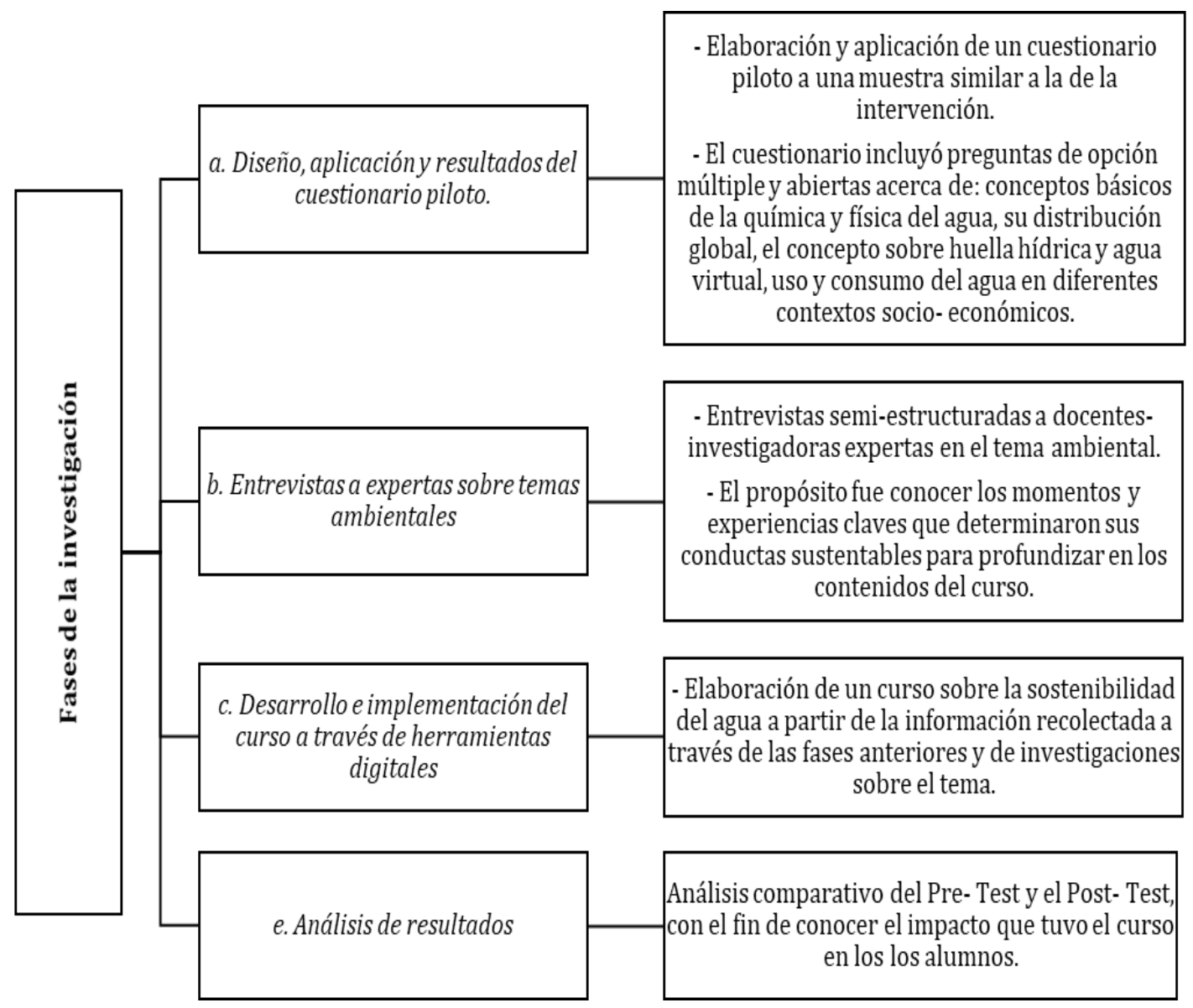

Figura 1. Fases de la investigación.

Para ello, se implementó un curso sobre la sostenibilidad del agua utilizando la plataforma de Canvas Instructure (2019). El curso constó de cuatro unidades, que incluían aspectos relevantes sobre temas relacionados con el agua desde un enfoque holístico con una mirada resolutiva. Las unidades fueron las siguientes: 1 . Elementos conceptuales sobre el agua; 2 . El agua y su importancia desde un enfoque holístico; 3. Situaciones y problemas actuales y futuros del agua y; 4 . Acciones como ciudadanos ambientales. Las unidades se impartieron en 4 sesiones, una sesión por semana con 
una duración de 3 horas cada una, en total 12 horas. Las sesiones se llevaron a cabo de manera presencial en su salón de clases dentro de la universidad. En cada sesión los alumnos acudieron con algún dispositivo móvil (laptop, Tablet o celular) que les permitió tener acceso a Internet y a la plataforma de Canvas Instructure (2019), que sirvió para la presentación del contenido del curso. Los alumnos trabajaron de forma individual y colectiva durante las sesiones, teniendo al docente como facilitador.

Antes de la implementación del curso se aplicó un cuestionario Pre-Test de 20 preguntas a través de la plataforma de Formularios Google, de las cuales, 9 fueron de respuestas abiertas y 11 de opción múltiple. Al final del curso se aplicó el Post-Test con las mismas preguntas para recabar el impacto que tuvo el curso en los conocimientos de los alumnos involucrados.

La dinámica de cada sesión se muestra a continuación en la figura 2.

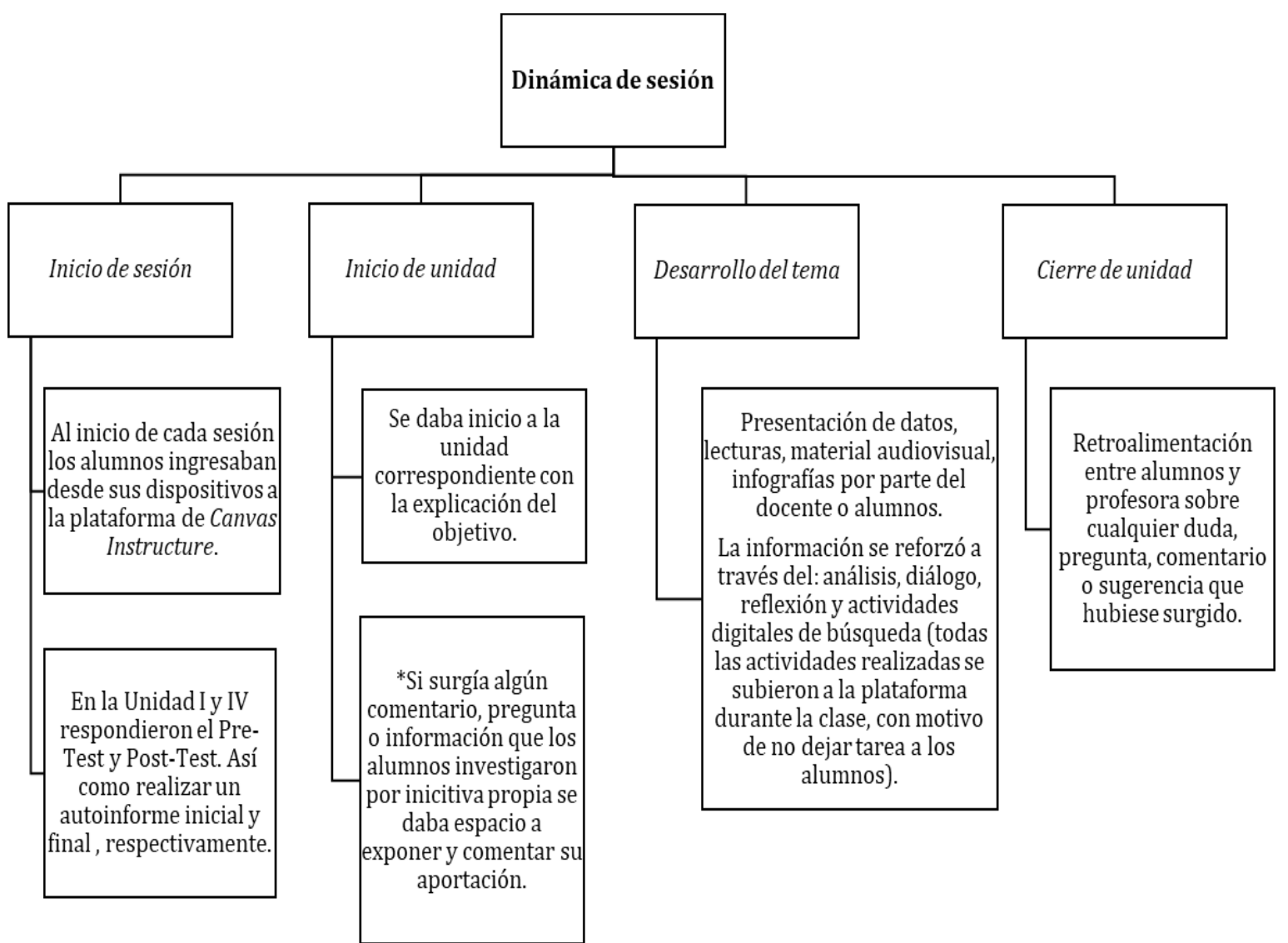

Figura 2. Dinámica que se realizó durante cada sesión del curso.

\section{Resultados y discusión}

Para analizar el impacto de la implementación del curso se compararon los porcentajes de respuesta obtenidos a cada pregunta del Pre-Test y del Post-Test. Dada la limitación de extensión del texto sólo se presentan algunos de los resultados obtenidos que nos parecieron relevantes. En todos los casos añadimos una breve descripción de los contenidos del curso que estuvieron relacionados con los temas y preguntas correspondientes.

Para comprender los resultados a la pregunta abierta sobre las consecuencias de extraer agua de los mantos acuíferos de una ciudad (tabla 1), se revisaron dentro del 
curso los siguientes elementos audiovisuales: La CDMX se hunde sobre los acuíferos que ha secado (Upixan, 2017); Escasez de Agua en México (Fuentes, 2016) y; Campaña Océanos-Cambio climático (SEMARNAT, 2018). Se puede apreciar en la tabla 1 que, durante el Pre-Test, el $41 \%$ de los participantes respondió que no conocía la respuesta, y el 48\% respondió escasez de agua en dichas zonas, en ambos casos respuestas no basadas en conocimientos documentados. Durante el Post-Test, el 48\% respondió: hundimiento del suelo debido al consumo de agua de los mantos acuíferos (que inicialmente era una respuesta con mínimos porcentajes). Hubo una disminución de la respuesta ligada a "escasez de agua en esas zonas", con un $27 \%$.

Se puede apreciar que los cambios en las respuestas están relacionados con los elementos audiovisuales y teóricos presentados por lo que, para los autores, resultó importante proporcionar esta información para que los universitarios comprendieran el alto riesgo que posee la ciudad de Querétaro, ciudad en la que viven, por agrietamientos y hundimientos y sus condiciones geográficas de semidesierto, (SIGEMA SA de CV., 2015). El desconocimiento de este tipo de temas provoca que las personas $\mathrm{y}$, en particular, los estudiantes de esta muestra, no comprendan las consecuencias de dañar o modificar el ambiente para la satisfacción de las necesidades humanas. Resulta fundamental incidir en la comprensión de los conocimientos sobre la utilización adecuada del recurso, con el fin de propiciar cambios de hábitos al hacer el uso del agua. Contreras et al. (2008) mencionan que la mayoría de las personas desgastan este recurso sin preocupación alguna, por lo que se requiere el tomar medidas acerca de este recurso invalorable y concientizar sobre su uso y preservación, de lo contrario se pueden originar y agudizar graves problemas. Esto ayuda a comprender la importancia de propiciar una alfabetización científica sobre estos temas y la importancia del agua subterránea en la escolaridad (Fernández, 2009). Al poseer esta nueva información, en el Post-Test se muestran respuestas que no se encontraron durante el Pre-Test, como la relación entre la escasez del agua y la afectación a la flora y fauna, así como el entendimiento de las consecuencias que existen al realizar extracciones desmesuradas de los mantos freáticos.

Tabla 1. Porcentaje de frecuencias de respuestas a la pregunta abierta sobre las consecuencias sobre la extracción de agua de mantos acuíferos, Pre-Test y Post-Test.

Pregunta: ¿Cuáles son las consecuencias de extraer el agua de los mantos acuíferos de una ciudad?

\begin{tabular}{lrc}
\hline Respuestas & Pre- Test & Post- Test \\
\hline Hundimiento del suelo debido al consumo de agua de los mantos acuíferos & $4 \%$ & $48 \%$ \\
\hline Escasez de agua en esas zonas & $41 \%$ & $27 \%$ \\
\hline Afectación de flora y fauna & $0 \%$ & $16 \%$ \\
\hline Inundaciones en zonas donde hay construcción y no permite la infiltración & $0 \%$ & $9 \%$ \\
\hline Se acaban los recursos & $7 \%$ & $0 \%$ \\
\hline Desbordes & $7 \%$ & $0 \%$ \\
\hline No sé & $41 \%$ & $0 \%$ \\
\hline
\end{tabular}

En la unidad II se revisó el tema del agua desde un enfoque holístico (geográfico, social, cultural, económico, político, ecológico, filosófico), por lo que a continuación se presenta 
una de las preguntas relacionadas a esta unidad. La tabla 2 presenta el porcentaje de respuestas ante la pregunta: el significado del concepto de Agua Virtual. Tema que se trató desde la información que ofrece la Comisión Nacional del Agua (CONAGUA, 2018) a partir de infografías y videos: El mundo tiene sed porque tenemos hambre (United Nations World Water Day, 2012a) y Todo lo que comes (United Nations World Water Day, 2012b), haciendo reflexión y análisis sobre el texto de Parada-Puig (2012) y Padilla et al. (2017).

Durante el Pre-Test se obtuvo que el 77\% de los alumnos respondió correctamente: "La cantidad total de agua que se requiere para obtener un producto". Durante el Post-Test el porcentaje a esta respuesta ascendió a un $96 \%$.

A través de la información proporcionada a los alumnos, entendieron que el consumo de agua no solo se realiza de manera directa, sino que también existe un consumo indirecto del cual no se percataban. El entender dicho concepto contribuyó a que conocieran el impacto de su huella hídrica y quisieran generar un cambio en su vida. Respecto a este tema se retomó el énfasis que Parada-Puig (2012) hace respecto a la enorme transferencia de agua a nivel mundial desde diversos productos (agua virtual), provocando que países exportadores se vean afectados por la escasez física o económica del agua, que, a su vez, hace que el costo del agua se eleve y crezca la contaminación. Por su parte, los países que importan productos van perdiendo seguridad alimentaria. Estos conceptos relacionados a la parte económica, ambiental y cultural fueron incluidos en el curso para que los participantes reflexionaran sobre la importancia del agua en el consumo de productos de consumo cotidiano.

Tabla 2. Porcentaje de frecuencia de respuestas a la pregunta de opción múltiple sobre el significado del concepto de Agua Virtual, Pre-Test y Post-Test.

\begin{tabular}{lcc}
\hline Pregunta: ¿Qué es el Agua Virtual? & & \\
\hline Respuestas & Pre-Test & Post-Test \\
\hline La cantidad total de agua que se requiere para obtener un producto & $77 \%$ & $96 \%$ \\
\hline Es la cantidad de agua que utilizamos en nuestra vida diaria & $8 \%$ & $4 \%$ \\
\hline Es el registro virtual de los cuerpos de agua que se conocen en el Planeta & $15 \%$ & $0 \%$ \\
\hline
\end{tabular}

La unidad IV enfocada a las Acciones como Ciudadanos Ambientales, revisó información sobre Educación Ambiental, Psicología Ambiental y Ciudadanía Ambiental, enfocada a las acciones que los alumnos podían realizar dentro de su cotidianidad; promoviendo la vinculación de la información de las unidades del curso. Se retomó el uso de infografías y videos. Los videos mostrados fueron los siguientes: Tu huella hídrica (hogar verde) (Vega, 2011), \#CaptaLaLluvia (IslaUrbana, 2016), Conciencia social y ahorro de agua (Correa, 2014), Comunidades Sustentable-Agua (Ministerio del Medio Ambiente, 2016).

En la pregunta abierta referente al comportamiento pro ambiental (tabla 3), se obtuvo el Pre-Test que el $45 \%$ de los alumnos lo asociaron a una conducta sustentable que busca el cuidado y protección ambiental; el 18\% respondió tener mayor educación y; el 5\% no supo la respuesta. Durante el Post-Test se obtuvo que el 57\% considera que el comportamiento pro ambiental ocurre a partir de una conducta sustentable que contenga determinadas virtudes y fortalezas a favor de la protección ambiental y; el $24 \%$ respondió que es necesario conocer las consecuencias del comportamiento humano para crear conciencia. 
Durante este tema los alumnos pudieron analizar los elementos audiovisuales mostrados, que les permitieron comprender que el comportamiento pro ambiental posee una gran relación con ciertos valores y fortalezas, como la responsabilidad, el compromiso, el altruismo, la empatía, el interés social, la sensibilidad, el respeto, la solidaridad y la integridad. Corral-Verdugo y de Quiroz (2004) mencionan que al poseer dicho comportamiento los seres humanos se vuelven virtuosos, ello a través de reflexionar, analizar y comprender las consecuencias de sus actos. Así mismo, la unión de los temas vistos durante el curso a través del diálogo y la reflexión logró que los alumnos pudieran entender este tipo de comportamiento en su día a día.

Tabla 3. Porcentaje de frecuencia de respuestas a la pregunta abierta sobre lo que se necesita para tener un Comportamiento Ambiental, Pre-Test y Post-Test.

\begin{tabular}{|c|c|c|}
\hline \multicolumn{3}{|l|}{ Pregunta: ¿Qué se necesita para tener un comportamiento pro ambiental? } \\
\hline Respuestas & Pre- Test & Post- Test \\
\hline $\begin{array}{l}\text { Conocer las consecuencias del comportamiento humano para crear } \\
\text { conciencia }\end{array}$ & $0 \%$ & $24 \%$ \\
\hline $\begin{array}{l}\text { Tener una conducta sustentable que busque el cuidado y protección } \\
\text { ambiental a partir de Valores y Fortalezas (responsabilidad, compromiso, } \\
\text { altruismo, empatía, interés social, sensibilidad, respeto, solidaridad, } \\
\text { integridad) }\end{array}$ & $45 \%$ & $57 \%$ \\
\hline Mayor educación & $18 \%$ & $13 \%$ \\
\hline Información de los temas & $10 \%$ & $4 \%$ \\
\hline Un equipo & $0 \%$ & $2 \%$ \\
\hline Conciencia & $16 \%$ & $0 \%$ \\
\hline Interés por el tema & $2 \%$ & $0 \%$ \\
\hline Reutilizar & $4 \%$ & $0 \%$ \\
\hline No sé & $5 \%$ & $0 \%$ \\
\hline
\end{tabular}

La tabla 4 muestra los resultados a la pregunta: definición del concepto de educación ambiental. Durante el Pre-Test, el 35\% hizo referencia al cuidado ambiental; el 31\% a crear conciencia sobre los recursos; y el 18\% no supo la respuesta. En el Post-Test, el $59 \%$ respondió que se trata de concientizar a las personas sobre su comportamiento y la importancia de los recursos mediante valores y fortalezas; el 26\% respondió que se trata de la correcta implementación de recursos para satisfacer necesidades de forma equilibrada; y el 15\% dijo que tiene que ver con un proceso de carácter comunitario, sistémico e interdisciplinario para informar y proteger el cuidado ambiental.

Durante el Pre-Test los alumnos pensaban que la Educación Ambiental solo comprendía de manera general el cuidado ambiental, pero mediante el curso pudieron comprender que dicho concepto incluye más elementos: interdisciplinarios, transdisciplinarios y multidisciplinarios. Siendo un concepto de suma importancia para los seres vivos y el ambiente. Navarro (2004) propone que la Educación Ambiental, en su más amplio sentido, permite promover las representaciones sociales del agua y otros temas ambientales. Por su parte, Calixto (2012) menciona que la Educación Ambiental no solo explica problemas del ambiente natural, sino también, del ambiente social y el ambiente transformado, por lo que esta unión hace evidente la necesidad de decidir y actuar sobre retos inmediatos sin perder de vista las acciones a mediano y largo plazo, por lo que la 
Educación Ambiental promueve la generación de nuevos comportamientos, actitudes, valores y creencias que impulsen nuevas relaciones entre los seres vivos y el ambiente.

Tabla 4. Porcentaje de frecuencia de respuestas de la pregunta abierta sobre el concepto de Educación Ambiental, Pre-Test y Post-Test.

Pregunta: Define qué es la Educación Ambiental

\begin{tabular}{lcc}
\hline Respuestas & Pre- Test & Post- Test \\
\hline $\begin{array}{l}\text { Concientizar a las personas sobre su comportamiento y la importancia } \\
\text { de los recursos mediante valores y virtudes }\end{array}$ & $0 \%$ & $59 \%$ \\
\hline $\begin{array}{l}\text { Proceso de carácter comunitario, sistémico e interdisciplinario para } \\
\text { informar, cuidar y promover el cuidado ambiental }\end{array}$ & $0 \%$ & $15 \%$ \\
\hline $\begin{array}{l}\text { Correcta implementación de los recursos para satisfacer necesidades de } \\
\text { forma equilibrada }\end{array}$ & $0 \%$ & $26 \%$ \\
\hline Utilizar y reutilizar recursos & $7 \%$ & $0 \%$ \\
\hline Cuidado ambiental & $35 \%$ & $0 \%$ \\
\hline Cambio climático & $3 \%$ & $0 \%$ \\
\hline Comprender la complejidad de la Naturaleza & $3 \%$ & $0 \%$ \\
\hline Crear conciencia & $31 \%$ & $0 \%$ \\
\hline Conocimiento de los recursos naturales & $3 \%$ & $0 \%$ \\
\hline No sé & $18 \%$ & $0 \%$ \\
\hline
\end{tabular}

La manera en que el curso se implementó alude a lo señalado por Mendieta y Gutiérrez (2014) sobre la importancia entre la Educación y la Tecnología para formar alternativas de solución ante la actual crisis ambiental por el agua y otros recursos ambientales, y crear una homogeneidad entre escuela y tecnología, así como el uso y consumo responsable de estos recursos en la educación formal e informal. Por su parte, Ruiz et al. (2009) sostienen que es necesario desarrollar estrategias didácticas, modelos de intervención (en el aula y en la sociedad) y procesos de simulación de la realidad que ofrezcan a los alumnos experiencias de aprendizaje efectivos mediante las que sean capaces de plantearse problemas y soluciones, discutir propuestas, planificar intervenciones, evaluar resultados y asumir posturas personales críticas sobre el tema.

\section{Conclusiones}

Considerando el objetivo general del estudio realizado, podemos decir que en general se encontró que la intervención realizada a través del curso sobre el tema de la sostenibilidad del agua mediante el uso de herramientas digitales, permitió apreciar una modificación en gran parte de los conceptos incluidos en el mismo, al comparar la situación del Pre-Test con la situación del Post-Test.

La participación de los estudiantes durante el curso posibilitó comprender el efecto que tuvo la información trabajada con las herramientas digitales (TIC) desde diversas disciplinas, así como el efecto del análisis, reflexión y crítica, sobre algunas actividades humanas que tienen relación con el agua y sustentabilidad, indicando el hecho de que promover el manejo de conocimiento más preciso favoreció la deconstrucción de prácticas y conceptos erróneos en los participantes. Así mismo, los resultados obtenidos permiten apreciar que la información ligada al cuidado del agua propicia un cambio de 
concepciones acerca de actitudes y comportamientos favorables hacia el ambiente, incidiendo así en la formación de la Ciudadanía Ambiental.

Nuestros resultados confirman los resultados obtenidos en estudios similares (Fernández et al., 2018) que han utilizado situaciones de aprendizaje utilizando TIC con diseños pre-test y pos test, en los que se genera mayor comprensión y conciencia ante los problemas ambientales. A este respecto, los participantes del presente estudio mencionaron que información anterior sobre el cuidado del agua que habían recibido en anteriores niveles educativos, había pasado desapercibida o se encontraba poco integrada a su contexto. La enseñanza de este tipo de información es necesaria para promover la comprensión de la situación global de manera holística (Diez-Martínez, 2013), así como la participación ciudadana, ya que guardan estrecha relación con la comprensión sobre la escasa cantidad de agua dulce a nivel mundial y su alta posibilidad de contaminación.

Gil y Vilches (2006) mencionan que la comprensión significativa de los conceptos exige superar el reduccionismo conceptual y plantear que la enseñanza de la Psicología y de la Educación Ambiental consista en actividades que integren aspectos conceptuales, procedimentales y axiológicos e, igualmente, una participación del ciudadano en el ambiente que lo rodea a él y a los demás seres del planeta. Por su parte, Osorio (2000), propone continuar con el avance del conocimiento ambiental al disminuir los efectos negativos que existen sobre él, además de fomentar la participación de las personas en procesos sociales que permitan cuestionar el tipo de ciencia y tecnología que se realiza en contra del ambiente, así como la creación de políticas públicas de ciencia y tecnología más acordes con las necesidades de una sociedad que busque la protección del ambiente a nivel global. Corres y Diez-Martínez (2019) proponen que mientras no se pueda concebir a través de la educación formal e informal al planeta como el gran patrimonio y bien común de todos los que habitamos en él, no podrá preservarse para las generaciones actuales ni futuras de los seres vivos, siendo esto la base de la construcción de la ciudadanía ambiental. De esta manera, la Educación Ambiental, en conjunto con la Psicología Ambiental, retoman elementos que pueden proporcionar una visión holística ante los seres vivos y el ambiente, velando por la importancia, el cuidado y la conservación del mundo en donde todo ocupa un papel relevante, por lo que el compromiso de las instituciones educativas, en todos los niveles educativos de la enseñanza y aprendizaje de estos temas, resulta fundamental para la conservación de los ecosistemas del planeta.

Estudios recientes (Edwards y Larson, 2020) indican que en nuestras sociedades actuales y más aún en aquellas prioritariamente urbanas, el interés y las posibilidades de tener contacto con la naturaleza de manera cotidiana ha disminuido, y las orientaciones de los jóvenes a este respecto van más de la biophilia a la videophilia. Ante esta situación, corresponde a los educadores el diseñar situaciones novedosas donde obtengan provecho de esta situación para atraer a los jóvenes a tener interés y respeto por su medio ambiente y todos los recursos y seres vivos del planeta.

Finalmente, si bien este tipo de experiencias educativas y de investigación, abren la discusión y las posibilidades a la generación de material que utilice las herramientas digitales de las que se dispone para la educación ambiental ciudadana, resulta necesario que este tipo de situaciones se reproduzcan nuevamente en otras poblaciones, otras edades, así como acerca de otras temáticas ambientales. 


\section{Agradecimientos}

Agradecemos al CONACYT el apoyo por una beca otorgada a la primera autora para realizar estudios de Maestría en Educación para la Ciudadanía.

\section{Referencias}

Calixto, R. (2012). Investigación en Educación Ambiental. Revista Mexicana de Investigación Educativa, 1019-1033. https://www.redalyc.org/articulo.oa?id=14024273002

Calvo, S., Ferreras, J., \& Rodrigo-Cano, D. (2020). La Educación Ambiental en las redes sociales: \#EA26. Revista de Educación Ambiental y Sostenibilidad, 2(1), 1-16. https://doi.org/10.25267/Rev_educ_ambient_sostenibilidad.2020.v2.i1.1301

Canvas Instructure (febrero, 2019). Canvas Instructure. [Página Web]. Recuperado de https://canvas.instructure.com/login/canvas

Carvalho de Sousa, A., Sevilla-Pavón, A., \& Seiz-Ortiz, R. (2012). Autonomy and ITC in Environmental Education. Procedia-Social and Behavioral Sciences, (46), 13431347.

Cervantes-Jiménez, M. C. (2017). Medición del costo de oportunidad de la exportación virtual de agua en los productos agrícolas mexicanos, 2004-2013. TANTALUS, Revista de economía de los recursos naturales, 1(1), 17-25.

CONAGUA.

(2018).

CONAGUA.

http://www.conagua.gob.mx/CONAGUA07/Contenido/Documentos/Infograf \%C3\%ADa\%20Huella\%20H\%C3\%ADdrica.pdf

Consejo Consultivo del Agua, A. C. (2018a). Agua en el mundo http://www.aguas.org.mx/sitio/index.php/panorama-del-agua/agua-en-elmundo

Consejo Consultivo del Agua, A. C. (2018b). Agua en México. http://www.aguas.org.mx/sitio/index.php/panorama-del-agua/agua-enmexico

Contreras, K., Contreras, J., Corti, M., De Sousa, J., Durán, M., \& Escalante, M. (2008). El agua un recurso para preservar. Mérida: Universidad de los Andes.

Corral-Verdugo, V., \& de Quiroz Pinheiro, J. (2004). Aproximaciones al estudio de la conducta sustentable. Medio Ambiente y Comportamiento Humano, 5(1-2),1-26.

Correa, L. (agosto, 2014). Conciencia social y ahorro de agua. [Archivo de video]. Recuperado en noviembre del 2018 de https://www.youtube.com/watch? $\mathrm{v}=$ ILnuJdRYOZM

Corres, A., \& Diez-Martínez, E. (2019). Efecto de un curso para promover actitudes pro-ambientales utilizando TIC: implicaciones para la educación en ciudadanía. En E. Diez-Martínez Day, Aproximaciones y perspectivas actuales en Educación para la Ciudadanía: aportes desde la intervención y la investigación (págs. 1-23). Eólica Grupo.

Diez-Martínez, E. (2013). Adolescents' Comprehension about Sustainable Development as a Device to Study Socio-Economic Knowledge Related to 
Citizenship. Citizenship, Social and Economics Education, 12(1), 18-29. https://doi.org/10.2304/csee.2013.12.1.18

Diez-Martínez, E. (2014). Lo esencial del Desarrollo Sustentable para jóvenes y no tan jóvenes. México: Fontamara.

Edwards, R. C., \& Larson, B. M. H. (2020). When screens replace backyards: strategies to connect digital-media-oriented young people to nature. Environmental Education Research, 26(7), 950-968, https://doi.org/10.1080/13504622.2020.1776844

Esparza, M. (2014). La sequía y la escasez de agua en México. Situación actual y $\begin{array}{lcc}\text { perspectivas futuras. } & \text { Secuencias } \\ \text { https://doi.org/10.18234/secuencia.v0i89.1231 }\end{array}$

Fernández Batanero, J. M., Reyes Rebollo, M. A., \& Piñero Virué, R. (2018). Design, application and evaluation of a technological instrument about environmental education. International journal of environmental and science education, 13(7), 579-588. https://hdl.handle.net/11441/79093

Fernández, G. (2009). El agua subterránea: estudio de esquemas de conocimiento en universitarios y estrategias didácticas para su aprendizaje significativo en estudiantes de secundaria. Granada: Universidad de Granada.

Fondo para la comunicación y la educación ambiental, A. C. (octubre, 2018). Visión general del Agua en México. AGUA.org.mx. https://agua.org.mx/cuanta-aguatiene-mexico/\#cuanta-hay

Fuentes, C. (mayo, 2016). Escasez de Agua en México. [Archivo de video]. Recuperado en diciembre del 2018 de https://www.youtube.com/watch? $\mathrm{v}=\mathrm{GIR} 24 \mathrm{PKQbDs} \& \mathrm{t}=1 \mathrm{~s}$

Galindo González, L. (2015). La educación ambiental en la virtualidad: un acercamiento al estado del arte. RIDE Revista Iberoamericana para la Investigación $y$ el Desarrollo Educativo, 5(10) 1-43. https://www.redalyc.org/articulo.oa?id=4981/498150318018

Gil Pérez, D., \& Vilches, A. (2006). Educación, ciudadanía y alfabetización científica: Mitos y Realidades. Revista Iberoamericana de Educación (42), 1-19. https://doi.org/10.35362/rie420760

Granados-Ramírez, R., \& Villaseñor-Franco, A. (2007). Entorno a la problemática del recurso del agua ante los megaproyectos de transporte. Estudio de caso Zona Metropolitana de la Ciudad de Querétaro. Boletín de la Sociedad Geológica Mexicana 59(2), 163-169. http://dx.doi.org/10.18268/BSGM2007v59n2a2

Gutiérrez-Yurrita, P. J. (2009). ¡A diseñar el futuro! El Holismo de la Tercera Cultura: hacia la integración científica y cultural. Centro de Estudios Jurídicos y Ambientales del Instituto Politécnico Nacional. http://doi.org/10.13140/RG.2.1.3788.0407

Gutiérrez-Yurrita, P. J. (2014). Ciudadanía Ambiental: retrospectiva histórica para reevolucionar su futuro. Aranzadi de Derecho Ambiental, (27), 37-59. 
Gwen, A. (2011). Enhancing college students' environmental sensibilities through online nature journaling. Environmental Education Research, 18(1), 133-150. https://doi.org/10.1080/13504622.2011.589000

Hines, J., Hungerford, H., \& Tomera, A. (1986). Analysis and synthesis of research on responsible behavior: a meta-analysis. The journal of Environmental Education, 18(2), 1-8. https://doi.org/10.1080/00958964.1987.9943482

INEGI (2020). Información de México para niños. Obtenido en octubre del 2020 de http://cuentame.inegi.org.mx/monografias/informacion/queret/poblacion/de fault.aspx?tema

IslaUrbana (enero, 2016). \#CaptaLaLluvia. [Archivo de video]. Recuperado enero del 2019 de https://www.youtube.com/watch?v=vpGZpvXM9z8

Kollmuss, A., \& Agyeman, J. (2002). Mind the Gap: Why do people act environmentally and what are the barriers to pro-environmental behavior? Environmental Education Research, 8(3), 239-260. https://doi.org/10.1080/13504620220145401

Mendieta, M. P., \& Gutiérrez, G. L. (2014). Actitudes ambientales hacia el agua, una exploración en estudiantes del municipio de Ventaquemada (Boyacá). Revista Luna Azul, (39), 40-62. http://www.scielo.org.co/pdf/luaz/n39/n39a04.pdf

Ministerio del Medio Ambiente. INDICAR PAÍS (enero, 2016). Comunidades Sustentable -Agua. [Archivo de video]. Recuperado en diciembre del 2018 de https://www.youtube.com/watch?v=PQYXq1HeFHQ

Naciones Unidas (1992). Declaración de Río sobre el Medio Ambiente y el Desarrollo. Obtenido en septiembre 2018 de https://www.un.org/spanish/esa/sustdev/documents/declaracionrio.htm

Naciones Unidas (2002). Cumbre de Johannesburgo 2002. Barcelona: Naciones Unidas.

Navarro, O. (2004). Representación social del agua y de sus usos. Psicología desde el Caribe, (14), 222-236. https://www.redalyc.org/pdf/213/21301409.pdf

Ochoa-Ramírez, J., Lamy, B., \& Serrano-Sánchez, Á. (2019). Migración interna y sus efectos en el crecimiento urbano del municipio de Querétaro. Quivera Revista

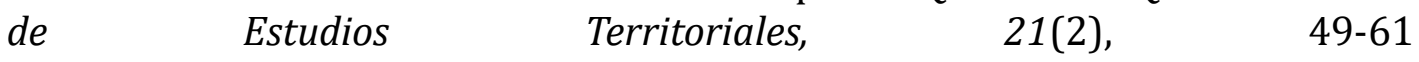
https://www.redalyc.org/jatsRepo/401/40161003004/html/index.html

Ojeda-Barceló, F., Gutiérrez-Pérez, J., \& Perales-Palacios. F. J. (2011). TIC y Sostenibilidad: Obstáculos y posibilidades para los educadores ambientales. Profesorado. Revista de Currículum y formación de Profesorado, 15(1), 263-313. http://hdl.handle.net/10481/15371

Osorio, C. M. (2000). Ética y Educación en Valores sobre el Medio Ambiente para el siglo XXI. Organización de Estados Iberoamericanos para la Educación, la Ciencia y la Cultura.

Padilla, A., Linos, J., Terrones, F., \& Rodríguez, Y. (2017). Conciencia estudiantil del agua virtual y su huella hídrica. Universidad Autónoma Metropolitana. http://doi.org/10.13140/RG.2.2.27109.50408 
Parada-Puig, G. (2012). El agua virtual: conceptos e implicaciones. Orinoquia, 16(1), 69-76. https://www.redalyc.org/articulo.oa?id=89625076001

Rodríguez-Peñaguirre, F., \& Rabell, E. (2019). Los derechos de los seres vivos y el ambiente. Una mirada desde el holismo. Metapolítica, (106), 39-52. https://comunicacion.buap.mx/sites/default/files/metapolitica106.pdf

Ruiz Mallén, I., Barraza, L., \& Ceja Adame, M. P. (2009). La educación para la sustentabilidad: análisis y perspectiva a partir de la experiencia de dos sistemas de bachillerato en comunidades rurales mexicanas. El Periplo Sustentable (16), 139-167.

Sauvé, L. (2005). Uma cartografia das corrientes em educaçao ambiental. En M. Sato, y I. Carvalho (Dirs.). Educação ambiental - Pesquisa e desafios. (pp. 17-46). Porto Alegre: Artmed. (En castellano, Cátedra de investigación de Canadá en educación ambiental. Université du Québec à Montréal). http://www.ecominga.uqam.ca/PDF/BIBLIOGRAPHIE/GUIDE_LECTURE_3/1/ 2.Sauve.pdf

Sauvé, L. (2014). Educación ambiental y ecociudadanía. Dimensiones claves de un proyecto político-pedagógico. Revista científica, (18), 12-23. Obtenido de https://revistas.udistrital.edu.co/index.php/revcie/article/view/5558/7072

SEMARNAT. (septiembre, 2018). Campaña Océanos Cambio climático. [Archivo de video]. Recuperado en enero del 2019 de https://youtu.be/Wxg2tsKcWwM

SIGEMA SA de CV. (2015). Atlas de Riesgos del Municipio de Querétaro 2015. Municipio de Querétaro. Querétaro: Protección Civil Querétaro.

United Nations World Water Day (2012)a. Todo lo que comes. [Archivo de video]. https://www.youtube.com/watch?v=0_z9cCe7wFs+2012

United Nations World Water Day (marzo, 2012)b. Día Mundial del Agua 2012 español El mundo tiene sed porque tenemos hambre. [Archivo de video]. Recuperado en noviembre del 2018 de https://www.youtube.com/watch?v=akmlrr3XyOo

Upixan. (marzo, 2017). La CDMX se hunde sobre los acuíferos que ha secado. [Archivo de video]. Recuperado en enero del 2019 de https://www.youtube.com/watch?v=1LXE9GPp1I0

Vega, 0. (noviembre, 2011). Tu huella hídrica (hogar verde). [Archivo de video]. Recuperado en diciembre del 2018 de https://www.youtube.com/watch? $\mathrm{v}=\mathrm{csOs} 4 \mathrm{VSifR} 4$

Vilches, A., \& Gil-Pérez, D. (2010). ¿Cómo puede contribuir la educación a la construcción de un futuro sostenible? Revista Eureka sobre Enseñanza y Divulgación de las Ciencias, 7(número extraordinario), 297-315. http://dx.doi.org/10.25267/Rev_Eureka_ensen_divulg_cienc.2010.v7.iextra.12

Yildirim, Z. (2005). Hypermedia as a Cognitive Tool: Student Teachers' Experiences in Learning by Doing. Educational Technology \& Society, 8(2), 107-117. 